\title{
MIMO Directional Modulation $M$-QAM Precoding for Transceivers Performance Enhancement
}

\author{
Ashkan Kalantari, Christos Tsinos, Mojtaba Soltanalian, \\ Symeon Chatzinotas, Wing-Kin Ma, and Björn Ottersten
}

\begin{abstract}
The increasing mobile data traffic requires network coverage expansion and rate enhancement. However, this demands more power and results in environmental pollution. As a solution, directional modulation can be used to provide efficient and interference-free communications. In this work, we geometrically model the extended detection regions of $M$-QAM modulation for $M=4,8,16,32$ and use these modeled regions to design energy efficient symbol-level precoders for an interferencefree MIMO directional modulation transceiver. We formulate and transform the precoder design problems into linearly constrained quadratic programming optimization problems. The simulation results show that compared with the benchmark schemes, directional modulation results in lower power consumption and symbol error rate using the less stringent extended detection regions and interference-free capability, respectively.
\end{abstract}

Keywords-Directional modulation, energy efficiency, M-QAM modulation, extended detection region, symbol-level precoding.

\section{INTRODUCTION}

According to Cisco [1], mobile data traffic will increase eight-fold from 2015 to 2020 . The extreme growth of video content and availability of mobile devices are the most important elements contributing to this growth. Orthogonal frequency division multiplexing access and time division multiplexing access [2], [3] are used to utilize the frequency and time resources to improve the rate of data communication. A multiple-input multiple-output (MIMO) system provides additional spatial degrees of freedom, though this comes at the cost of interference that appears among the transmitted streams. To better use the spatial freedom, a MIMO transceiver needs to deal with the inter-stream interference via pre/postprocessing techniques. As a more advanced approach to enhance the communications, preprocessing at the transmitter is applied to use the spatial dimension in multiple-antenna transmitters [4], [5]. Recently, there has been interest in directional modulation [6]-[9] and constructive interference [10]-[13] techniques which can initiate interference-free or interferencelimited communications in a MIMO system. In the directional modulation, the antenna weights are designed such that the radio frequency signals get modulated after passing through the channel and result in the desired symbol at the desired antenna. In directional modulation, it is possible to design the precoder such that the communicated symbol is exactly the desired

\footnotetext{
This work was supported by the National Research Fund (FNR) of Luxembourg under AFR grant for the project SeMIGod. Ashkan Kalantari, Christos Tsinos, Symeon Chatzinotas, and Björn Ottersten are with the Interdisciplinary Centre for Security, Reliability and Trust (SnT), The University of Luxembourg, 29, avenue JF Kennedy, L-1855 Luxembourg-Kirchberg, Luxembourg, (E-mails: \{ashkan.kalantari,christos.tsinos,symeon.chatzinotas,bjorn.ottersten\} @uni.lu) Mojtaba Soltanalian is with the Department of Electrical and Computer Engineering, University of Illinois at Chicago, Chicago, IL 60607, E-mail: (msol@uic.edu). Wing-Kin $\mathrm{Ma}$ is with the Department of Electronic Engineering, The Chinese University of Hong Kong, Shatin, Hong Kong, China (e-mail:wkma@ieee.org).
}

symbol or it is close to the desired symbol [9]. The former case can be translated into interference-free and the latter case can be translated into communication with interference among the data streams.

Apart from data demand, mobile communications consumes a large amount of energy and has a considerable share in environmental pollution [14]. Reducing the energy consumed in the radio access networks is not only environmental friendly, but also decreases the communications cost of both the operators and users. The researches in [9]-[11], [15], [16] study the relaxed design in constructive interference and directional modulation to improve the energy efficiency at the transmitter.

In this work, we use the concept of directional modulation to jointly address the data rate and energy efficiency issue of mobile communications. The works of [9]-[11], [15], [16] focus on $M$-PSK precoder design and [13] designs the constructive interference precoder for $M$-QAM when $M=16$, however, there is no work on designing the directional modulation precoder for $M$-QAM with $M=4,8,16,32$, while utilizing the extended detection regions of these constellations. To fill this gap, we design an energy efficient symbol-level precoder for an $M$-QAM directional modulation MIMO transceiver. In addition, we propose compact geometrical characterization for the extended detection regions of the mentioned $M$ QAM modulations where the minimum Euclidean distance between the symbols is kept as in the conventional $M$-QAM constellation, which can be translated into interference-free communication. In this design, each symbol is placed in the optimal location of the constellation, hence, less power is consumed to communicate in a specific rate. This means that the available power can be used to communicate with a higher rate.

The remainder of this paper is organized as follows. In Section II, the signal model is introduced. In Section III, the extended detection regions of various $M$-QAM modulations are geometrically characterized, and then the precoder design problem is formulated and transformed into a standard form. In Section IV, we present simulation results by comparing the proposed method with the benchmark schemes. Finally, we draw the conclusions in Section V.

Notation: Upper-case and lower-case bold-faced letters are used to denote matrices and column vectors, respectively. The superscripts $(\cdot)^{T},(\cdot)^{*}$, and $(\cdot)^{H}$ represent transpose, conjugate, and Hermitian operators, respectively. $\mathbf{I}_{N \times N}$ denotes an $N$ by $N$ identity matrix, $\mathbf{a} \circ \mathbf{b}$ is the element-wise Hadamard product, $\mathbf{a}^{\circ n}$ is the element-wise Hadamard power, $\mathbf{0}$ is the all zero vector, $\mathbf{1}$ is the all unit vector, $\|\cdot\|$ is the Frobenius norm, $|\cdot|$ represents the absolute value of a scalar, and $\operatorname{Card}(\cdot)$ shows the number of elements in a set. $\operatorname{Re}(\cdot), \operatorname{Im}(\cdot)$, and $\arg (\cdot)$ represent the real part, imaginary part, and angle of a complex 
number, respectively.

\section{Signal AND SyStem Model}

In our model, a single-carrier directional modulation transmitter equipped with $N_{t}$ antennas, denoted by $T$, communicates with a receiver having $N_{r}$ antennas, denoted by $R$, using $M$-QAM modulation where $M=4,8,16,32$. The received signal, $\mathbf{y}$, at $R$ is

$$
\mathbf{y}=\mathbf{H w}+\mathbf{n},
$$

where $\mathbf{y}$ is an $N_{r} \times 1$ vector denoting the received signals by $R, \mathbf{H}=\left[\mathbf{h}_{1}, \ldots, \mathbf{h}_{n}, \ldots, \mathbf{h}_{N_{r}}\right]^{T}$ is an $N_{r} \times N_{t}$ matrix denoting the channel from $T$ to $R, \mathbf{h}_{n}$ is an $N_{t} \times 1$ vector containing the channel coefficients from the transmitter antennas to the $n$-th antenna of $R$, and $\mathbf{w}$ is the transmit vector containing the antenna weights. The random variable $\mathbf{n} \sim$ $\mathcal{C N}\left(\mathbf{0}, \sigma^{2} \mathbf{I}_{N_{r} \times N_{r}}\right)$ denotes the additive white Gaussian noise at $R$, where $\mathcal{C} \mathcal{N}^{r}$ denotes a complex and circularly symmetric random variable. In directional modulation, the elements of $\mathbf{H w}=\left[s_{1}^{\prime}, \ldots, s_{n}^{\prime}, \ldots, s_{N_{r}}^{\prime}\right]^{T}$ are the induced $M$-QAM symbols on the antennas of $R$ where $s_{n}^{\prime}$ is the induced $M$-QAM symbol on the $n$-th antenna of $R, \mathbf{s}=\left[s_{1}, \ldots, s_{n}, \ldots, s_{N_{r}}\right]$ are the $M$-QAM symbols to be communicated between $T$ and $R$. For each modulation, the power of the symbols is divided by the average symbol power, e.g., 10 wat for 16-QAM, of the corresponding modulation to have statistically average unit power for the communicated symbols, i.e., $\mathbb{E}\left[\left|s_{i}\right|^{2}\right]=1$. $R$ can apply conventional detectors on each antenna to detect the symbols. In the next section, we design the optimal precoders for the mentioned $M$-QAM constellations.

\section{CharacterizATION OF EXTENDED DETECTION REGIONS AND OPTIMAL PRECODER DESIGN}

In this section, first, we geometrically characterize the extended detection regions for $M$-QAM modulation where $M=4,8,16,32$. Then, we use the characterized regions to formulate and design the corresponding optimal directional modulation precoders for the mentioned modulation orders.

\section{A. Characterization of the Freedom Regions}

In this part, we derive analytical expressions to concisely characterize the extended detection regions, defined by solid regions and dashed lines, of the $M$-QAM constellations shown in Fig. 1. To begin, we divide each constellation into multiple sets, as illustrated in Figures 1(a) to 1(d), and model the extended detection region for each set of symbols.

1) The case of $M=4$ : Assume that $s_{n}$ is a symbol which we need to communicate with the receiver. The extended detection region of the symbol in the first quadrant, $s_{n} \in \mathfrak{s}_{1_{4}}$, of 4-QAM constellation in Fig. 1(a) can be modeled as

$$
\operatorname{Re}\left(\mathbf{h}_{n}^{T} \mathbf{w}\right) \geq \sqrt{\gamma} \operatorname{Re}\left(s_{n}\right), \operatorname{Im}\left(\mathbf{h}_{n}^{T} \mathbf{w}\right) \geq \sqrt{\gamma \operatorname{Im}}\left(s_{n}\right),
$$

where $\gamma=10^{S N R / 10}$ is the minimum required amplification power for the induced symbol and $S N R$ shows the minimum required signal to noise ratio in $\mathrm{dB}$ at the corresponding receiving antenna. The defined extended detection regions in (2) cannot be used in other constellation quadrants since satisfying the amplification level for negative values of real or/and imaginary parts of $s_{n}$ changes the direction of the inequalities. To solve this, both sides of the inequalities in (2) can be multiplied by $\operatorname{Re}\left(s_{n}\right)$ and $\operatorname{Im}\left(s_{n}\right)$ to make both sides positive. Note that this does not change the direction of inequalities since $\operatorname{Re}\left(s_{n}\right)$ and $\operatorname{Im}\left(s_{n}\right)$ have the same sign as $\operatorname{Re}\left(\mathbf{h}_{n}^{T} \mathbf{w}\right)$ and $\operatorname{Im}\left(\mathbf{h}_{n}^{T} \mathbf{w}\right)$, respectively, at the optimal point of precoder design. Hence, the extended detection region for any 4-QAM symbol of Fig. 1(a), $s_{n} \in \mathfrak{s}_{1_{4}}$, can be characterized using the following expression

$$
\begin{aligned}
& \operatorname{Re}\left(s_{n}\right) \operatorname{Re}\left(\mathbf{h}_{n}^{T} \mathbf{w}\right) \geq \sqrt{\gamma} \operatorname{Re}^{2}\left(s_{n}\right), \\
& \operatorname{Im}\left(s_{n}\right) \operatorname{Im}\left(\mathbf{h}_{n}^{T} \mathbf{w}\right) \geq \sqrt{\gamma} \operatorname{Im}^{2}\left(s_{n}\right) .
\end{aligned}
$$

2) The case of $M=8$ : The extended detection regions for the symbols $s_{n} \in \mathfrak{s}_{1_{s}}$ can be characterized in the same way as in (3). The extended detection region for the upper and lower symbols of $s_{n} \in \mathfrak{s}_{2}$ can be respectively modeled using the following expressions

$$
\begin{aligned}
& \operatorname{Re}\left(\mathbf{h}_{n}^{T} \mathbf{w}\right)=\sqrt{\gamma} \operatorname{Re}\left(s_{n}\right), \operatorname{Im}\left(\mathbf{h}_{n}^{T} \mathbf{w}\right) \geq \sqrt{\gamma} \operatorname{Im}\left(s_{n}\right), \\
& \operatorname{Re}\left(\mathbf{h}_{n}^{T} \mathbf{w}\right)=\sqrt{\gamma} \operatorname{Re}\left(s_{n}\right), \operatorname{Im}\left(\mathbf{h}_{n}^{T} \mathbf{w}\right) \leq \sqrt{\gamma} \operatorname{Im}\left(s_{n}\right) .
\end{aligned}
$$

The characterizations of $\mathfrak{s}_{2}$ in (4) and (5) can be mixed to get a unified expression as

$$
\operatorname{Re}\left(\mathbf{h}_{n}^{T} \mathbf{w}\right)=\sqrt{\gamma} \operatorname{Re}\left(s_{n}\right), \operatorname{Im}\left(s_{n}\right) \operatorname{Im}\left(\mathbf{h}_{n} \mathbf{w}\right) \geq \sqrt{\gamma} \operatorname{Im}^{2}\left(s_{n}\right) .
$$

3) The case of $M=16$ : For $s_{n} \in \mathfrak{s}_{1_{16}}$ of 16-QAM constellation in Fig. 1(c), the extended detection region can be modeled the same as in (3). Also, the extended detection region for the symbols $s_{n} \in \mathfrak{s}_{2}$ can be modeled in the same way as (6). The extended detection region for the right and left hand symbols of $s_{n} \in \mathfrak{s}_{3}$ can be modeled as

$$
\begin{aligned}
& \operatorname{Re}\left(\mathbf{h}_{n}^{T} \mathbf{w}\right) \geq \sqrt{\gamma} \operatorname{Re}\left(s_{n}\right), \operatorname{Im}\left(\mathbf{h}_{n}^{T} \mathbf{w}\right)=\sqrt{\gamma} \operatorname{Im}\left(s_{n}\right), \\
& \operatorname{Re}\left(\mathbf{h}_{n}^{T} \mathbf{w}\right) \leq \sqrt{\gamma} \operatorname{Re}\left(s_{n}\right), \operatorname{Im}\left(\mathbf{h}_{n}^{T} \mathbf{w}\right)=\sqrt{\gamma} \operatorname{Im}\left(s_{n}\right),
\end{aligned}
$$

which can be compressed into

$$
\operatorname{Re}\left(s_{n}\right) \operatorname{Re}\left(\mathbf{h}_{n}^{T} \mathbf{w}\right) \geq \sqrt{\gamma} \operatorname{Re}^{2}\left(s_{n}\right), \operatorname{Im}\left(\mathbf{h}_{n}^{T} \mathbf{w}\right)=\sqrt{\gamma} \operatorname{Im}\left(s_{n}\right) .
$$

In the case $s_{n} \in \mathfrak{s}_{4}$, the symbols needs to be modeled as fixed points using (10) in order to keep the standard Euclidean distance to neighborhood symbols.

$$
\operatorname{Re}\left(\mathbf{h}_{n}^{T} \mathbf{w}\right)=\sqrt{\gamma} \operatorname{Re}\left(s_{n}\right), \operatorname{Im}\left(\mathbf{h}_{n}^{T} \mathbf{w}\right)=\sqrt{\gamma} \operatorname{Im}\left(s_{n}\right) .
$$

4) The case of $M=32$ : The extended detection regions of the symbols $s_{n} \in \mathfrak{s}_{2}, s_{n} \in \mathfrak{s}_{3}$ and $s_{n} \in \mathfrak{s}_{4}$ can be modeled using the expressions developed in (6), (9), and (10), respectively. To model the extended detection regions for the symbols in the sets $\mathfrak{s}_{5}$ and $\mathfrak{s}_{6}$ of Fig. 1(d), first, we model the extended detection regions for the symbols $s_{n} \in \mathfrak{s}_{5}$ and $s_{n} \in \mathfrak{s}_{6}$ in the first quadratic and then rotate the symbols of other quadrants into the first quadrant to apply the defined extended detection region in the first quadrant on them. The extended detection regions for the symbols $s_{n} \in \mathfrak{s}_{5}$ and $s_{n} \in \mathfrak{s}_{6}$ of the first quadrant can be geometrically modeled using the following expressions

$$
\begin{aligned}
& 3 \sqrt{\gamma} \leq \operatorname{Im}\left(\mathbf{h}_{n}^{T} \mathbf{w}\right) \leq \operatorname{Re}\left(\mathbf{h}_{n}^{T} \mathbf{w}\right)-2 \sqrt{\gamma} \\
& \operatorname{Re}\left(\mathbf{h}_{n}^{T} \mathbf{w}\right)+2 \sqrt{\gamma} \leq \operatorname{Im}\left(\mathbf{h}_{n}^{T} \mathbf{w}\right), \operatorname{Re}\left(\mathbf{h}_{n}^{T} \mathbf{w}\right) \geq 3 \sqrt{\gamma} .
\end{aligned}
$$




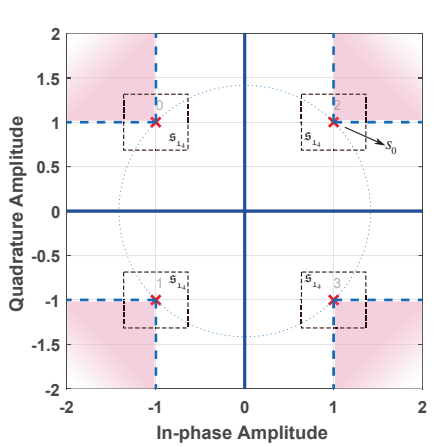

(a) Extended detection regions for 4-QAM constellation.

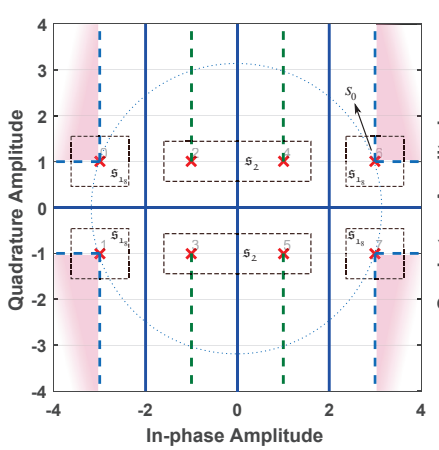

(b) Extended

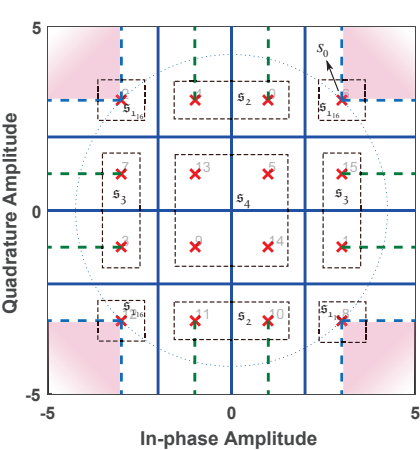

(c) Extended detection

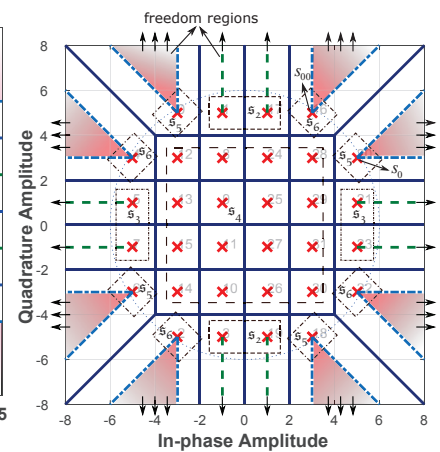

(d) Extended detection regions for 32QAM constellation.

Fig. 1: Characterization of relaxed detection regions for $M$-QAM constellations. The numbers show the decimal equivalent of the gray code for each symbol. The extended detection regions are shown by dashed lines and solid regions for $M=4,8,16,32$.

Now, we multiply the symbols $s_{n} \in \mathfrak{s}_{5}$ and $s_{n} \in \mathfrak{s}_{6}$ by $e^{i \varphi_{n_{5}}}$ and $e^{i \varphi_{n_{6}}}$, respectively, so that they rotate into the extended detection regions of the first quadrant defined in (11) and (12). Here, $\varphi_{n_{5}}$ is the phase difference between $s_{0}$ of Fig. 1(d) and $s_{n} \in \mathfrak{s}_{6}$, and $\varphi_{n_{6}}$ is the phase difference between $s_{00}$ of Fig. 1(d) and $s_{n} \in \mathfrak{s}_{6}$.

\section{B. Optimal Precoder Design}

In this section, we use the characterized extended detection regions in Section III-A to formulate and design the optimal $M$-QAM MIMO directional modulation precoders when $M=$ $4,8,16,32$.

The optimal precoder design for 4-QAM is formulated as

$$
\begin{aligned}
\min _{\mathbf{w}} & \|\mathbf{w}\|^{2} \\
\text { s.t. } & \operatorname{Re}\left(s_{n_{1}}\right) \operatorname{Re}\left(\mathbf{h}_{n_{1}}^{T} \mathbf{w}\right) \geq \sqrt{\gamma} \operatorname{Re}^{2}\left(s_{n_{1}}\right), s_{n_{1}} \in \mathfrak{s}_{1_{4}} \\
& \operatorname{Im}\left(s_{n_{1}}\right) \operatorname{Im}\left(\mathbf{h}_{n_{1}}^{T} \mathbf{w}\right) \geq \sqrt{\gamma} \operatorname{Im}^{2}\left(s_{n_{1}}\right) .
\end{aligned}
$$

We can cast the optimal precoder design for 8-QAM as

$$
\begin{aligned}
\min _{\mathbf{w}} & \|\mathbf{w}\|^{2} \\
\text { s.t. } & \Omega_{1}\left(s_{n_{1}}\right), s_{n_{1}} \in \mathfrak{s}_{1_{8}} \\
& \operatorname{Re}\left(\mathbf{h}_{n_{2}}^{T} \mathbf{w}\right)=\sqrt{\gamma} \operatorname{Re}\left(s_{n_{2}}\right), s_{n_{2}} \in \mathfrak{s}_{2} \\
& \operatorname{Im}\left(s_{n_{2}}\right) \operatorname{Im}\left(\mathbf{h}_{n_{2}} \mathbf{w}\right) \geq \sqrt{\gamma} \operatorname{Im}^{2}\left(s_{n_{2}}\right) .
\end{aligned}
$$

where $\Omega_{1}\left(s_{n_{1}}\right)$ represents the constraints of design problem (13). Next, we can formulate the optimal precoder design problem for 16-QAM as follows:

$$
\begin{array}{ll}
\min _{\mathbf{w}} & \|\mathbf{w}\|^{2} \\
\text { s.t. } & \Omega_{1}\left(s_{n_{1}}\right), s_{n_{1}} \in \mathfrak{s}_{1_{1} 6} \\
& \Omega_{2}\left(s_{n_{2}}\right), s_{n_{2}} \in \mathfrak{s}_{2} \\
& \operatorname{Re}\left(s_{n_{3}}\right) \operatorname{Re}\left(\mathbf{h}_{n_{3}}^{T} \mathbf{w}\right) \geq \sqrt{\gamma} \operatorname{Re}^{2}\left(s_{n_{3}}\right), s_{n_{3}} \in \mathfrak{s}_{3} \\
& \operatorname{Im}\left(\mathbf{h}_{n_{3}}^{T} \mathbf{w}\right)=\sqrt{\gamma} \operatorname{Im}\left(s_{n_{3}}\right) . \\
& \operatorname{Re}\left(\mathbf{h}_{n_{4}}^{T} \mathbf{w}\right)=\sqrt{\gamma} \operatorname{Re}\left(s_{n_{4}}\right), s_{n_{4}} \in \mathfrak{s}_{4} \\
& \operatorname{Im}\left(\mathbf{h}_{n_{4}}^{T} \mathbf{w}\right)=\sqrt{\gamma} \operatorname{Im}\left(s_{n_{4}}\right) .
\end{array}
$$

where $\Omega_{2}\left(s_{n_{2}}\right)$ represents the constraints (14b) and (14c). Finally, the optimal precoder design problem for $32-\mathrm{QAM}$ is defined as

$$
\begin{array}{ll}
\min _{\mathbf{w}} & \|\mathbf{w}\|^{2} \\
\text { s.t. } & \Omega_{2}\left(s_{n_{2}}\right), s_{n_{2}} \in \mathfrak{s}_{2} \\
& \Omega_{3}\left(s_{n_{3}}\right), s_{n_{3}} \in \mathfrak{s}_{3} \\
& \Omega_{4}\left(s_{n_{4}}\right), s_{n_{4}} \in \mathfrak{s}_{4} \\
& 3 \sqrt{\gamma} \leq \operatorname{Im}\left(\widetilde{\mathbf{h}}_{n_{5}}^{T} \mathbf{w}\right) \leq \operatorname{Re}\left(\widetilde{\mathbf{h}}_{n_{5}}^{T} \mathbf{w}\right)-2 \sqrt{\gamma}, s_{n_{5}} \in \mathfrak{s}_{5} \\
& \operatorname{Re}\left(\widetilde{\mathbf{h}}_{n_{6}}^{T} \mathbf{w}\right)+2 \sqrt{\gamma} \leq \operatorname{Im}\left(\widetilde{\mathbf{h}}_{n_{6}}^{T} \mathbf{w}\right), s_{n_{6}} \in \mathfrak{s}_{6} \\
& \operatorname{Re}\left(\widetilde{\mathbf{h}}_{n_{6}}^{T} \mathbf{w}\right) \geq 3 \sqrt{\gamma},
\end{array}
$$

where $\widetilde{\mathbf{h}}_{n_{5}}^{T}=\mathbf{h}_{n_{5}}^{T} e^{i \varphi_{n_{5}}}, \widetilde{\mathbf{h}}_{n_{6}}^{T}=\mathbf{h}_{n_{6}}^{T} e^{i \varphi_{n_{6}}}, \Omega_{3}\left(s_{n_{3}}\right)$ represents the constraint (15c) and (15d), $\Omega_{4}\left(s_{n_{4}}\right)$ represents the constraints (15e) and (15f). Here, we proceed with transforming (16) into a standard form. A similar approach can be applied to the design problems in (13) to (15). To simplify (16), first, we stack the constraints of (16) as

$$
\begin{array}{cl}
\min _{\mathbf{w}} & \|\mathbf{w}\|^{2} \\
\text { s.t. } & \operatorname{Re}\left(\mathbf{H}_{2} \mathbf{w}\right)=\mathbf{s}_{r e_{2}}, \operatorname{Im}\left(\mathbf{S}_{2}\right) \operatorname{Im}\left(\mathbf{H}_{2} \mathbf{w}\right) \geq \mathbf{s}_{i m_{2}}^{\circ 2}, \\
& \operatorname{Re}\left(\mathbf{S}_{3}\right) \operatorname{Re}\left(\mathbf{H}_{3} \mathbf{w}\right) \geq \mathbf{s}_{r e_{3}}^{\circ 2}, \operatorname{Im}\left(\mathbf{H}_{3} \mathbf{w}\right)=\mathbf{s}_{i m_{3}}, \\
& \operatorname{Re}\left(\mathbf{H}_{4} \mathbf{w}\right)=\mathbf{s}_{r e_{4}}, \operatorname{Im}\left(\mathbf{H}_{4} \mathbf{w}\right)=\mathbf{s}_{i m_{4}} . \\
& 3 \times \mathbf{1}_{5} \leq \operatorname{Im}\left(\widetilde{\mathbf{H}}_{5} \mathbf{w}\right) \leq \operatorname{Re}\left(\widetilde{\mathbf{H}}_{5} \mathbf{w}\right)-2 \times \mathbf{1}_{5} \\
& \operatorname{Re}\left(\widetilde{\mathbf{H}}_{6} \mathbf{w}\right)+2 \times \mathbf{1}_{6} \leq \operatorname{Im}\left(\widetilde{\mathbf{H}}_{6} \mathbf{w}\right) \\
& \operatorname{Re}\left(\widetilde{\mathbf{H}}_{6} \mathbf{w}\right) \geq 3 \times \mathbf{1}_{6},
\end{array}
$$

where $\mathbf{s}_{r e_{j}}$ and $\mathbf{s}_{i m_{j}}$ are the vectors that respectively stack the real and imaginary parts of the symbols $s_{n_{j}} \in \mathfrak{s}_{j}$ multiplied by $\sqrt{\gamma}, \mathbf{1}_{j}$ is a $\operatorname{Card}\left(\mathfrak{s}_{\mathfrak{j}}\right) \times 1$ vector with elements multiplied by $\sqrt{\gamma}$, and $\mathbf{S}_{j}$ is a diagonal matrix with diagonal entries as $s_{n_{j}} \in \mathfrak{s}_{j}$. Next, we proceed to remove the real and imaginary parts from (17). Similar as in [9], we have

$$
\operatorname{Re}\left(\mathbf{H}_{j} \mathbf{w}\right)=\mathbf{H}_{j a} \widetilde{\mathbf{w}}, \operatorname{Im}\left(\mathbf{H}_{j} \mathbf{w}\right)=\mathbf{H}_{j b} \widetilde{\mathbf{w}},
$$


where $\widetilde{\mathbf{w}}=\left[\operatorname{Re}\left(\mathbf{w}^{T}\right), \operatorname{Im}\left(\mathbf{w}^{T}\right)\right]^{T}, \quad \mathbf{H}_{j a}=$ $\left[\operatorname{Re}\left(\mathbf{H}_{j}\right),-\operatorname{Im}\left(\mathbf{H}_{j}\right)\right], \mathbf{H}_{j b}=\left[\operatorname{Im}\left(\mathbf{H}_{j}\right), \operatorname{Re}\left(\mathbf{H}_{j}\right)\right]$, and $\|\widetilde{\mathbf{w}}\|^{2}=\|\mathbf{w}\|^{2}$. Using (18), we can reformulate (17) as

$$
\begin{array}{cl}
\min _{\mathbf{w}} & \|\mathbf{w}\|^{2} \\
\text { s.t. } & \mathbf{H}_{2 a} \mathbf{w}=\mathbf{s}_{r e_{2}}, \operatorname{Im}\left(\mathbf{S}_{2}\right) \mathbf{H}_{2 b} \mathbf{w} \geq \mathbf{s}_{i m_{2}}^{\circ 2}, \\
& \operatorname{Re}\left(\mathbf{S}_{3}\right) \mathbf{H}_{3 a} \mathbf{w} \geq \mathbf{s}_{r e_{3}}^{\circ 2}, \mathbf{H}_{3 b} \mathbf{w}=\mathbf{s}_{i m_{3}}, \\
& \mathbf{H}_{4 a} \mathbf{w}=\mathbf{s}_{r e_{4}}, \mathbf{H}_{4 b} \mathbf{w}=\mathbf{s}_{i m_{4}}, \\
& 3 \times \mathbf{1}_{5} \leq \widetilde{\mathbf{H}}_{5 b} \mathbf{w} \leq \widetilde{\mathbf{H}}_{5 a} \mathbf{w}-2 \times \mathbf{1}_{5}, \\
& \widetilde{\mathbf{H}}_{6 a} \mathbf{w}+2 \times \mathbf{1}_{6} \leq \widetilde{\mathbf{H}}_{6 b} \mathbf{w}, \widetilde{\mathbf{H}}_{6 a} \mathbf{w} \geq 3 \times \mathbf{1}_{6},
\end{array}
$$

Stacking the constraints of (19) yields

$$
\min _{\mathbf{w}}\|\mathbf{w}\|^{2} \text { s.t. } \quad \mathbf{A} \mathbf{w} \geq \mathbf{a}, \mathbf{B w}=\mathbf{b}
$$

where

$$
\begin{aligned}
\mathbf{A} & =\left(\begin{array}{l}
\operatorname{Im}\left(\mathbf{S}_{2}\right) \mathbf{H}_{2 b} \\
\operatorname{Re}\left(\mathbf{S}_{3}\right) \mathbf{H}_{3 a} \\
\widetilde{\mathbf{H}}_{5 a}-\widetilde{\mathbf{H}}_{5 b} \\
\widetilde{\mathbf{H}}_{5 b} \\
\widetilde{\mathbf{H}}_{6 b}-\widetilde{\mathbf{H}}_{6 a} \\
\widetilde{\mathbf{H}}_{6 a}
\end{array}\right), \mathbf{a}=\left(\begin{array}{l}
\mathbf{s}_{i m_{2}}^{\circ 2} \\
\mathbf{s}_{r e_{3}}^{\circ 2} \\
2 \times \mathbf{1}_{5} \\
3 \times \mathbf{1}_{5} \\
2 \times \mathbf{1}_{6} \\
3 \times \mathbf{1}_{6}
\end{array}\right), \\
\mathbf{B} & =\left(\begin{array}{l}
\mathbf{H}_{2 a} \\
\mathbf{H}_{3 b} \\
\mathbf{H}_{4 a} \\
\mathbf{H}_{4 b}
\end{array}\right), \mathbf{b}=\left(\begin{array}{l}
\mathbf{s}_{r e_{2}} \\
\mathbf{s}_{i m_{3}} \\
\mathbf{s}_{r e_{4}} \\
\mathbf{s}_{i m_{4}}
\end{array}\right) .
\end{aligned}
$$

As we see, (20) is a linearly constraint quadratic optimization problem, which is convex and can be solved using standard optimization techniques.

\section{Simulation Results}

In this section, we demonstrate the performance of the proposed method and compare it with the benchmark schemes. As the performance metrics, we use the average power consumption and symbol error rate (SER) over multiple designed precoders and channel realizations. In all simulations, channels are considered to be quasi static block Rayleigh fading, which are generated using i.i.d. complex Gaussian random variables with distribution $\mathcal{C N}(0,1)$ and remain fixed during the communication of a group of $N_{r} M$-QAM symbols. Also, the noise is generated using i.i.d. complex Gaussian random variables with distribution $\mathcal{C} \mathcal{N}\left(0, \sigma^{2}\right)$. We use the acronyms "DM" and "Opt LP" in the legend of the figures instead of the terms "directional modulation" and "optimal linear precoding". Here, we assume that adaptive coding and modulation is used, hence, each modulation order is used in a specific SNR range. First, we mention the two benchmark schemes and then proceed with the simulation scenarios.

We consider zero-forcing (ZF) at the transmitter [17] and optimal linear precoding [18] as benchmark schemes since both of them use the CSI knowledge at the transmitter to combat interference. The received signal at $R$ in $\mathrm{ZF}$ scheme is $\mathbf{y}=\mathbf{H W} \mathbf{s}+\mathbf{n}$ where $\mathbf{W}=\mathbf{H}^{H}\left(\mathbf{H H}^{H}\right)^{-1}$ is the precoding vector and vector $\mathbf{s}$ contains the symbols to be transmitted.
The optimal linear precoding design problem is [18]

$$
\min _{\mathbf{w}_{1}, \ldots, \mathbf{w}_{N_{r}}} \sum_{i=1}^{N_{r}}\left\|\mathbf{w}_{i}\right\|^{2} \text { s.t. } \frac{\left|\mathbf{h}_{k}^{T} \mathbf{w}_{k}\right|^{2}}{\sum_{j \neq k}^{N_{r}} \mathbf{h}_{j}^{T} \mathbf{w}_{j}+\sigma_{j}^{2}} \geq \gamma, k=1, \ldots, N_{r} .
$$

For the first scenario, we measure the transmitter's consumed power versus $N_{t}$ for the proposed and benchmark schemes, shown in Fig. 2. It is observed that the proposed designs consume considerably less power than ZF and optimal linear precoding benchmark schemes. This difference increases as the modulation order decreases. For example, 4-QAM and 8QAM schemes almost consume $6 \mathrm{~dB}$ and $5 \mathrm{~dB}$ lower power compared to optimal linear precoding when $N_{t}=N_{r}=10$. This is due to the fact that in contrast to the benchmark, directional modulation places each symbol in the optimal location of the constellation. Particularly, 4-QAM has the most difference in power consumption with the benchmark scheme since all the 4-QAM constellation points have extended detection regions.

In the second scenario, we measure the average SER versus the minimum required SNR at the receiver for the proposed and benchmark schemes in Fig. 3. Note that the transmission power of the benchmark schemes does not depend on the modulation order since the precoder depends only on the channel and the average transmit power of the symbols is unit. First, we see that the proposed scheme provides lower SER compared to ZF in relatively low SNR regimes, particularly for $M=4,8$. The reason is that though both the proposed scheme and ZF result in interference-free communication, due to optimal symbol-level design, the SNR of the communicated symbol in the proposed scheme can go above the minimum required value, especially for $N_{t}=N_{r}$. Second, the difference between the SER of the proposed method and the optimal linear precoding increases as the SNR goes higher. This is due to the fact that the communication using optimal linear precoding results in interference and as SNR increases, the effect of interference increases. On the other hand, directional modulation using the extended detection regions with standard Euclidean distance results in interference-free communication.

By comparing Figures 2 and 3 for $N_{t}=N_{r}$, it is seen that the proposed scheme results in both power reduction at the transmitter and SER reduction at the receiver. These gains come at the price of higher complexity requirement at the transmitter to design the precoder for each group of symbols.

\section{Conclusions}

In this work, the extended detection regions of $M$-QAM modulation for $M=4,8,16,32$ were geometrically modeled and these models were used to design the optimal symbol-level precoder for a MIMO directional modulation transmitter to communicate interference-free $M$-QAM symbols. In contrast to the conventional ZF and optimal linear precoding benchmark schemes, it was observed that directional modulation consumes less power at the transmitter. Also, it results in lower SER at the receiver compared to the benchmark schemes due to interference-free communication capability and the possibility to induce symbols with a SNR higher than the required threshold. Directional modulation provides power and SER reduction since it places the symbols in the optimal location of the extended detection region of each constellation. 


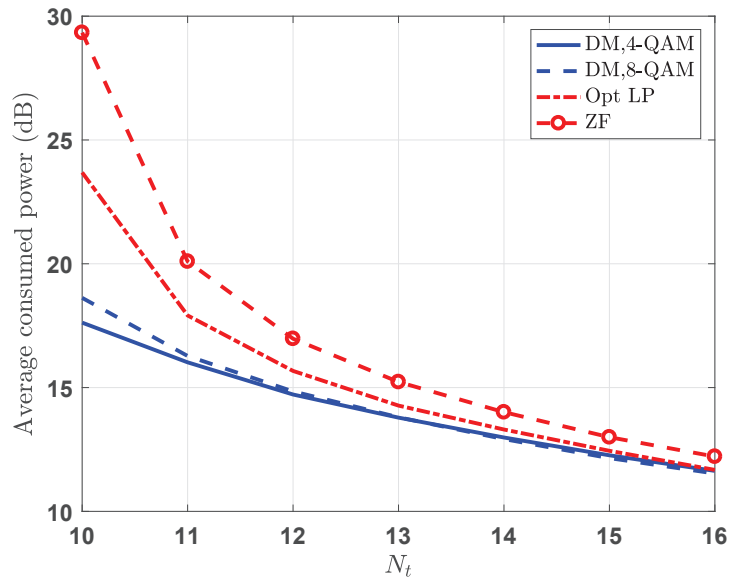

(a) $S N R=10 \mathrm{~dB}$.



(b) $S N R=20 \mathrm{~dB}$.

Fig. 2: Average total consumed power with respect to $N_{t}$ for the proposed M-QAM directional modulation precoding and the benchmark schemes when $N_{r}=10$.



Fig. 3: Average SER with respect to the required SNR at the receiver for the proposed $M$-QAM directional modulation precoding and the benchmark schemes when $N_{t}=N_{r}=10$.

\section{REFERENCES}

[1] "Cisco visual networking index: Forecast and methodology, 20152020," Cisco Systems, Tech. Rep., 2015.

[2] S. Weinstein and P. Ebert, "Data transmission by frequency-division multiplexing using the discrete fourier transform," IEEE Trans. Commun. Technol., vol. 19, no. 5, pp. 628-634, Oct. 1971.

[3] D. D. Falconer, F. Adachi, and B. Gudmundson, "Time division multiple access methods for wireless personal communications," IEEE Commun. Mag., vol. 33, no. 1, pp. 50-57, Jan. 1995.

[4] Q. Spencer, A. Swindlehurst, and M. Haardt, "Zero-forcing methods for downlink spatial multiplexing in multiuser MIMO channels," IEEE Trans. Signal Process., vol. 52, no. 2, pp. 461-471, Feb. 2004.

[5] N. Sidiropoulos, T. Davidson, and Z.-Q. Luo, "Transmit beamforming for physical-layer multicasting," IEEE Trans. Signal Process., vol. 54 no. 6, pp. 2239-2251, Jun. 2006.

[6] A. Babakhani, D. Rutledge, and A. Hajimiri, "Transmitter architectures based on near-field direct antenna modulation," IEEE J. Solid-State Circuits, vol. 43, no. 12, pp. 2674-2692, Dec. 2008.

[7] M. Daly and J. Bernhard, "Directional modulation technique for phased arrays," IEEE Trans. Antennas Propag., vol. 57, no. 9, pp. 2633-2640, Sep. 2009.

[8] M. Daly, E. Daly, and J. Bernhard, "Demonstration of directional modulation using a phased array," IEEE Trans. Antennas Propag., vol. 58, no. 5, pp. 1545-1550, May 2010.

[9] A. Kalantari, M. Soltanalian, S. Maleki, S. Chatzinotas, and B. Ottersten, "Directional modulation via symbol-level precoding: A way to enhance security," IEEE J. Sel. Topics Signal Process., vol. PP, no. 99, Aug. 2016.

[10] C. Masouros and E. Alsusa, "Dynamic linear precoding for the exploitation of known interference in MIMO broadcast systems," IEEE Trans. Wireless Commun., vol. 8, no. 3, pp. 1396-1404, Mar. 2009.

[11] _ - "Soft linear precoding for the downlink of DS/CDMA communication systems," IEEE Trans. Veh. Technol., vol. 59, no. 1, pp. 203-215, Jan. 2010.

[12] M. Alodeh, S. Chatzinotas, and B. Ottersten, "Constructive multiuser interference in symbol level precoding for the MISO downlink channel," IEEE Trans. Signal Process., vol. 63, no. 9, pp. 2239-2252, May 2015.

[13] _ "Constructive interference through symbol level precoding for multi-level modulation," in IEEE Global Commun. Conf. (GLOBECOM), CA, San Diego, Dec. 2015.

[14] A. Fehske, G. Fettweis, J. Malmodin, and G. Biczok, "The global footprint of mobile communications: The ecological and economic perspective," IEEE Commun. Mag., vol. 49, no. 8, pp. 55-62, Aug. 2011

[15] C. Masouros and G. Zheng, "Exploiting known interference as green signal power for downlink beamforming optimization," IEEE Trans. Signal Process., vol. 63, no. 14, pp. 3628-3640, Jul. 2015.

[16] M. Alodeh, S. Chatzinotas, and B. Ottersten, "Energy-efficient symbollevel precoding in multiuser MISO based on relaxed detection region," IEEE Trans. Wireless Commun., vol. 15, no. 5, pp. 3755-3767, May 2016

[17] L.-U. Choi and R. Murch, "A transmit preprocessing technique for multiuser MIMO systems using a decomposition approach," IEEE Trans. Wireless Commun., vol. 3, no. 1, pp. 20-24, Jan. 2004.

[18] M. Bengtsson and B. Ottersten, Handbook of Antennas in Wireless Communications. CRC Press, 2001, ch. Optimal and suboptimal transmit beamforming, pp. 18-1-18-33. 